\title{
A PROPOSED METHOD FOR IMPROVING THE PERFORMANCE OF P-TYPE GaAs IMPATT
}

\author{
H. A. El-Motaafy \\ EE Dep., High Technology Institute (HTI), Ramadan-Tenth City, Cairo, Egypt
}

\begin{abstract}
A special waveform is proposed and assumed to be the optimum waveform for p-type GaAs IMPATTs. This waveform is deduced after careful and extensive study of the performance of these devices. The results presented here indicate the superiority of the performance of the IMPATTs driven by the proposed waveform over that obtained when the same IMPATTs are driven by the conventional sinusoidal waveform. These results are obtained using a full-scale computer simulation program that takes fully into account all the physical effects pertinent to IMPATT operation. In this paper, it is indicated that the superiority of the proposed waveform is attributed to its ability to reduce the bad effects that usually degrade the IMPATT performance such as the space-charge effect and the drift-velocity dropping below saturation effect. The superiority is also attributed to the ability of the proposed waveform to improve the phase relationship between the terminal voltage and the induced current.
\end{abstract}

Key Words: Computer-Aided Design, GaAs IMPATT, Microwave Engineering

\section{INTRODUCTION}

It is well known that the performance of n-type GaAs IMPATT diodes is superior to that of p-type diodes ${ }^{[1-3]}$. This is attributed mainly to the velocity-field characteristic of electrons in the GaAs material that has a negative-differential mobility region ${ }^{[1-3]}$. This allows the electrons to have higher velocities at the lower values of field encountered in the active region during the negative half cycle of operation. This is in contrast to holes whose velocity drops below the saturated drift velocity for the lower values of the field. Since these effects are inherent in GaAs material, it is only possible to improve the performance of p-type GaAs IMPATTs through modifying the microwave circuit where the diode is imbedded. Since it has been previously reported that the performance of IMPATTs could be improved if it is driven by a non-sinusoidal voltage waveform ${ }^{[4-6]}$, the external circuit attached to the diode must be capable of supporting such waveforms. However, no comprehensive study has been conducted for determining the optimum waveform. In this paper, a systematic procedure for choosing the optimum waveform and designing the external microwave circuit is presented. A special waveform is proposed to be the optimum waveform for p-type GaAs IMPATT. This proposition is supported by an extensive investigation of
IMPATT operation. This investigation has been conducted using a full-scale computer simulation program that takes fully into account all the physical effects pertinent to IMPATT operation without resorting to approximations that limits the scope of its application. This program is a modified version of one described elsewhere ${ }^{[7]}$.

The performance of a given IMPATT that is expected to be modest is studied when it is driven by this waveform and when it is driven by the conventional sinusoidal waveform. Then the results obtained for the two cases are compared. The superiority of the proposed waveform (PWF) is demonstrated especially at higher values of the dc current density $J_{d c}$. The PWF can be optimized further through optimizing its parameters. Since the PWF is difficult to be realized practically, the performance of the IMPATT is studied when it is driven by the waveform $V_{2}$. This waveform comprises only the first two harmonics of the proposed waveform. The performance in this case is found also to be much superior to the conventional case.

\section{THE PROPOSED WAVEFORM}

The proposed waveform $\left(\mathrm{V}_{\mathrm{F}}\right)$ is shown in Fig. 1 for the case where $A_{2}=90 \mathrm{~V}$. In the first part of the cycle, $\phi_{1}$, the height of the PWF is $A_{1}$. In the second part, $\phi_{2}$, the height is $A_{2}$. In the negative half cycle the height is $A_{3}$. The width of the transitions between the different parts is $\varepsilon_{1}, \varepsilon_{2}$, and $\varepsilon_{3}$. The waveform consisting of the first two harmonics of the proposed waveform denoted by $V_{2}$ is shown in the same figure together with the conventional waveform $\left(\mathrm{V}_{\mathrm{C}}\right)$. The parameters of this waveform can be optimized according to the following guidelines that are deduced from our extensive study of IMPATT operation ${ }^{[1,2,7-10]}$ :

1. $\varepsilon_{1}$ should be as small as possible to reduce the spread of transit times of charge carriers.

2. $\varepsilon_{2}$ should be as small as possible to increase the slope of the terminal voltage when crossing the breakdown level. This helps reduce the space charge effect and improve the phase delay provided by the avalanche process.

3. $A_{1}$ should be null to reduce the power dissipation at the beginning of the RF cycle.

4. The choice of $\phi_{2}$ and $A_{2}$ is a compromise between the following factors:

a. $\phi_{2}$ should be minimized in order to reduce the spread of transit times of carriers. 
b. $\mathrm{A}_{2}$ must be increased to reduce the space charge effect and the spread of transit times of carriers and to satisfy the breakdown condition. However, $\mathrm{A}_{2}$ must not be too high, lest the breakdown condition should be satisfied only by an excessive increase of the dc-breakdown voltage. This is attributed to the saturation of the ionization rates at the high values of electric field.

c. The product $\phi_{2} \mathrm{~A}_{2}$ must be as high as possible to enable the increase of $A_{3}$, which improves the performance.

5. The choice of $\phi_{3}$ and $A_{3}$ is a compromise between the following factors:

a. $A_{3}$ must be sufficiently high in order to extract the maximum energy from the drifting carriers but must not be too high so as to cause the carrier drift velocity to drop below the saturated value.

b. $\phi_{3}$ must be reduced to delay the injection of the avalanche-generated packet (AGP) in the drift region; but if $\phi_{3}$ is excessively reduced, the conductance of the diode may become positive at some higher order harmonics. The effect of the parameter $\mathrm{A}_{2}$, and consequently $\mathrm{A}_{3}$, will be presented in this paper.

\section{THE RESULTS OBTAINED FOR THE CHOSEN IMPATT}

The IMPATT whose performance will be studied is a GaAs p-type one having a single-drift abrupt junction structure. The doping density in the drift region is $5 \times 10^{15} \mathrm{~cm}^{-3}$ and its width is $3.7 \mu \mathrm{m}$.

The PWF has the following parameters:

$\varepsilon_{1}=\varepsilon_{2}=\varepsilon_{3}=9^{\circ}, \Phi_{1}=72^{\circ}, \Phi_{2}=90^{\circ}$,

$\Phi_{3}=171^{\circ}, A_{1}=90 \mathrm{~V}$, and $\mathrm{A}_{2}=49.5 \mathrm{~V}$.

Figures 2 and 3 show, respectively, the efficiency and the RF power density versus the dc current density $J_{d c}$ for the PWF and a sinusoidal signal of amplitude $70 \mathrm{~V}$. The value of $70 \mathrm{~V}$ is chosen since it gives the best performance for the sinusoidal excitation at the highest values of $J_{d c}$ considered.

Figures 4 and 5 show the efficiency and the RF power for the waveform $\mathrm{V}_{2}$ consisting only of the first two harmonics of the PWF. The results indicate clearly the superiority of the proposed waveforms over the conventional sinusoidal signal especially at the higher values of the dc bias current where the efficiency becomes almost three times higher and the RF power six times higher for the PWF. This demonstrates the ability of the proposed waveforms to reduce considerably the space-charge effect. This is illustrated clearly in Fig. 6, which shows the induced current $J_{i}$ for the proposed waveform and the sinusoidal signal at different values of the dc bias current for the PWF and the sinusoidal signal. It is clear that the major manifestation of the reduction of the space charge effect is the delay of the injection of the avalanche-generated packet in the drift region (DR). This means that the time delay provided by the avalanche process is increased. This contributes to the improvement of the performance. The performance is also improved by reducing the spread of the transit times of carriers. This causes the AGP and consequently the peak of $\mathrm{J}_{\mathrm{i}}$ to be sharper, as the figures show. Hence, $\mathrm{J}_{\mathrm{i}}$ has higher values for the PWF during the negative half cycle. This is attributed also to the reduction of the drift velocity dropping below the saturation (VDBS) effect. On the other hand, the shape and the values of the induced current in the negative half cycle are better for the proposed waveforms. This is attributed to the reduction of the spread of transit times of carriers for the PWFs. The reduction of the spread of transit times of carriers means that the generated pulse of holes will be more localized in both the time domain and the space. This is clearly demonstrated in Fig. 7 that shows the spatial distribution of the hole current density for the considered waveforms at different phase angles. As it is seen at the beginning of the RF cycle, some carriers from the previous cycle are still being extracted at the ohmic contact. The extraction of these carriers is carried out earlier for $\mathrm{V}_{\mathrm{F}}$. Hence, the start of the avalanche breakdown is considerably delayed for the proposed waveforms. This contributes to their better performance since the phase delay provided by the avalanche process is enhanced. The injection of the AGP of holes is also delayed for the proposed waveform. Correspondingly, the phase delay is increased. This effect considerably improves the performance. As the AGP of holes drifts toward the ohmic contact it is dispersed by the effect of diffusion. This dispersion is enhanced by the spacecharge effect for $\mathrm{V}_{\mathrm{C}}$. For $\mathrm{V}_{\mathrm{F}}$, the dispersion is smaller. This is attributed to two facts: that the AGP is sharper in both the space and the time domain, and the spacecharge effect is less significant for the PWF. The reduction of the spread of transit times means that the currents induced by the carriers have a better distribution of phase angles. Consequently, the induced current will be higher. The drift-velocity dropping below saturation is less significant for $\mathrm{V}_{\mathrm{F}}$. This causes almost all the holes to drift at the saturated velocity. Hence, the induced current is higher and more flat for this waveform. This contributes to its better performance.

Figures 8 and 9 show the spatial distribution of the electron current $\left(\mathrm{J}_{\mathrm{n}}\right)$, and the spatial distribution of the electric field $\mathrm{E}$ at different phase angles for different values of dc current. By analyzing these results, it is clear that the AGP, after being injected into the drift region, causes $E$ to decrease behind it and to increase in its front. This effect becomes more significant as $J_{d c}$ increases. The decrease of the field behind the AGP causes the avalanche generation to be suppressed early in the cycle, which reduces the spread of the transit times of the carriers and causes the peak of $\mathrm{J}_{\mathrm{i}}$ to be sharper. Hence, the performance is improved. The increase of the field in the front of the AGP suppresses the VDBS effect. Hence, $J_{i}$ will have higher values during the negative half cycle and the efficiency is enhanced. The figures show also that $J_{i}$ is extremely 
small during the major portion of the positive half cycle for the PWF. This is attributed to the shape of the PWF and helps improve the efficiency.

Figures 10 and 11 show the effect of A2 on the performance of the p-type IMPATT. The increase of both the RF power and the efficiency with $A_{2}$ is attributed mainly to the reduction of the space charge 


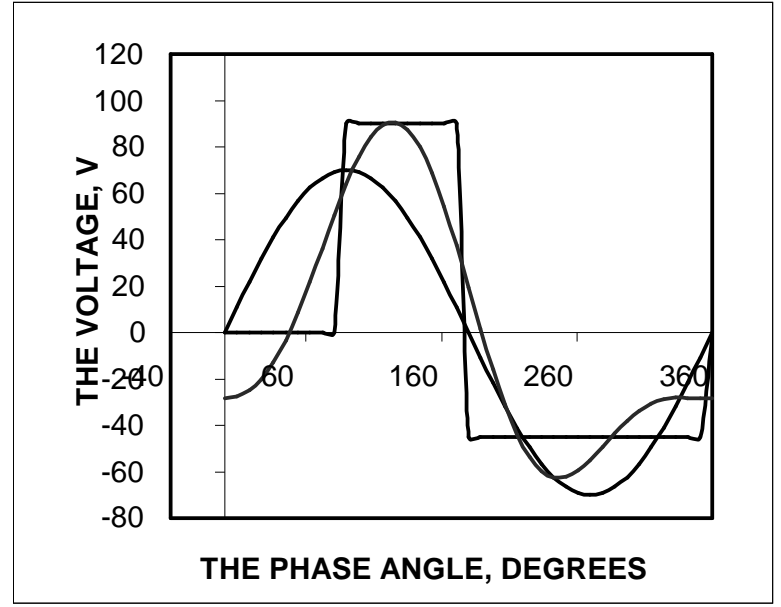

Fig.1 The voltage waveforms VC, VF, and VS

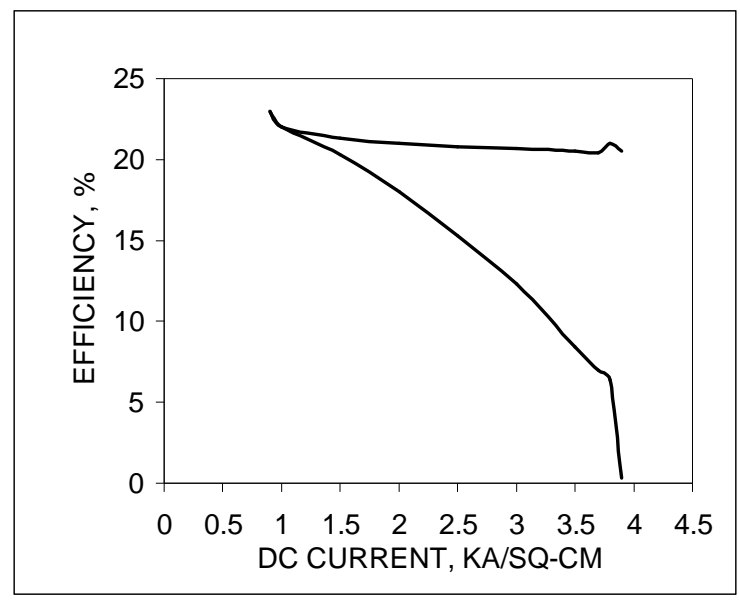

Fig. 2 The efficiency versus DC current for $V_{F}$ and $V_{C}$ at $F=9$ $\mathrm{G} \mathrm{Hz}$

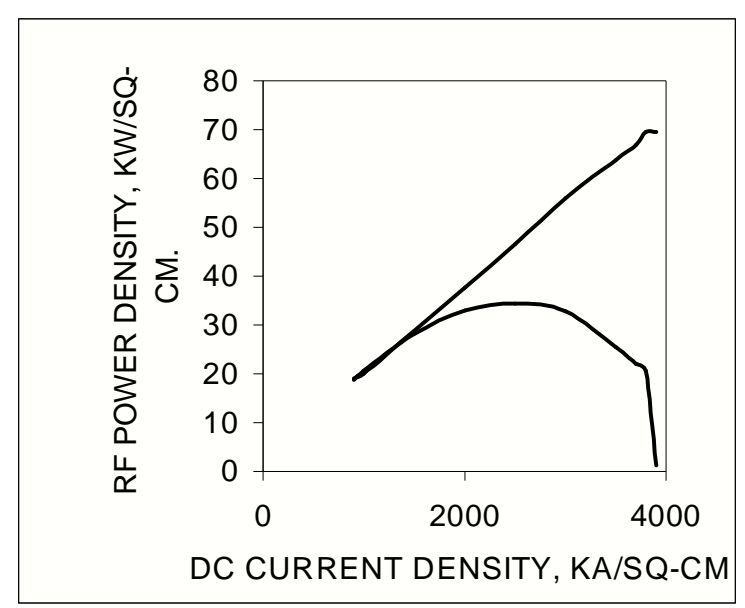

Fig. 3 The RF power versus DC current for $V_{F}$ and $V_{C}$

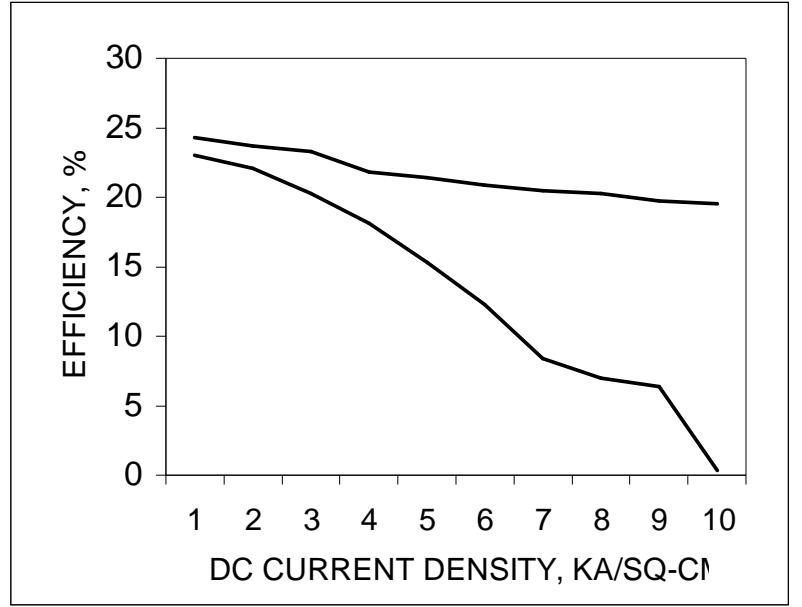

Fig. 4 The efficiency versus DC current density

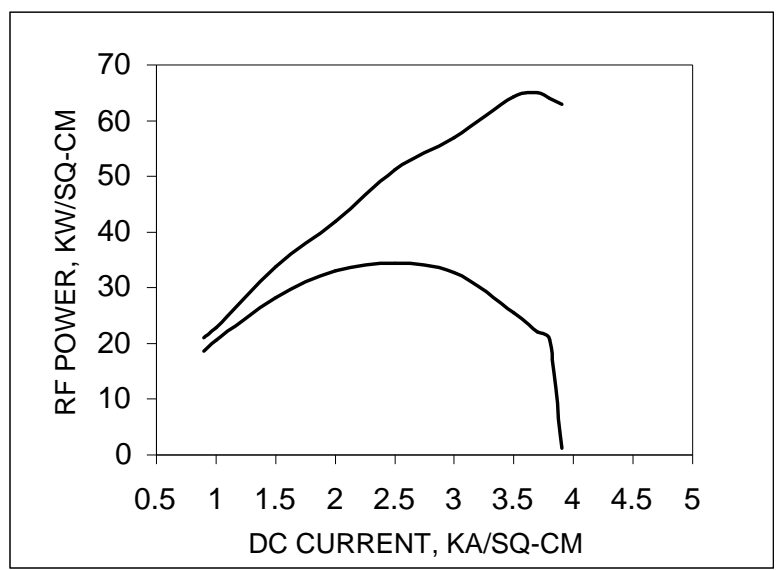

Fig. $5 \mathrm{RF}$ power versus $\mathrm{DC}$ current for $\mathrm{V}_{2}$ and $\mathrm{V}_{\mathrm{C}}$ at $\mathrm{F}=9 \mathrm{G}$ $\mathrm{Hz}$

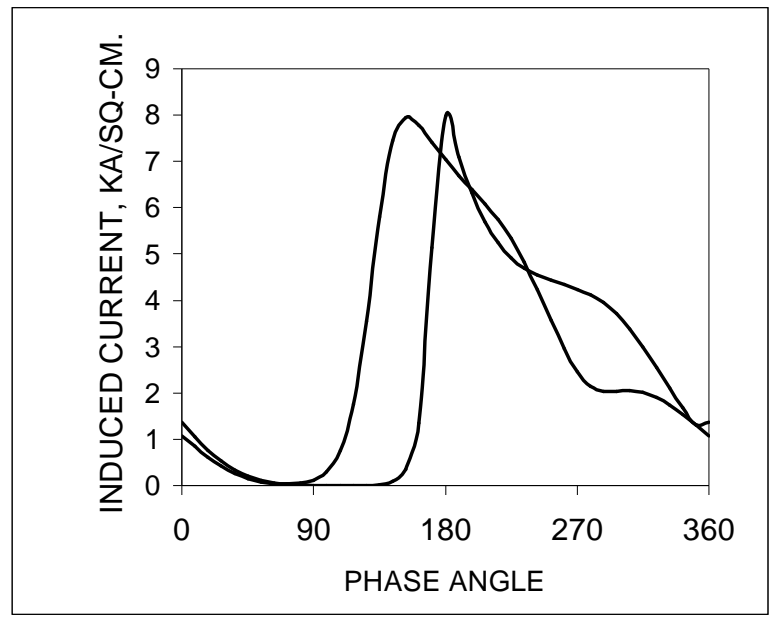

Fig. 6A The induced current for $V_{F}$ and $V_{C}$ at $J_{D C}=3 \mathrm{KA} / \mathrm{cm}^{2}$ 


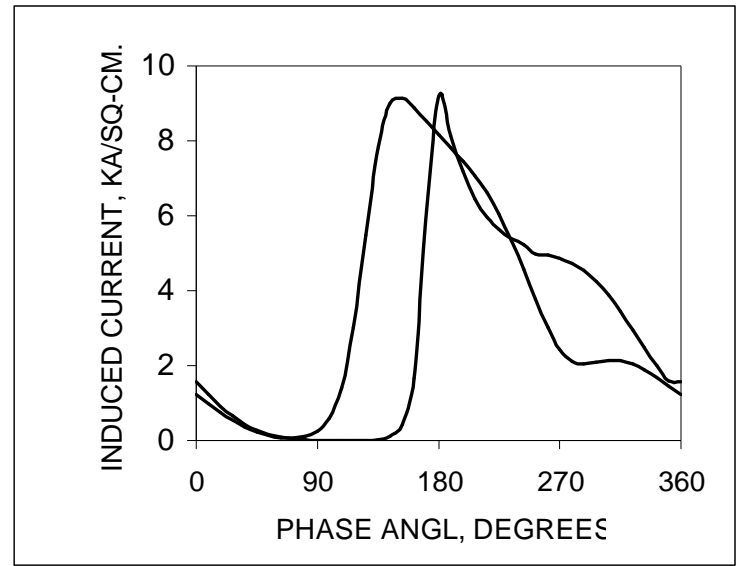

Fig. 6B The induced current for $\mathrm{VF}$ and $\mathrm{VC}$ at JDC $=3.5$ $\mathrm{KA} / \mathrm{cm} 2$

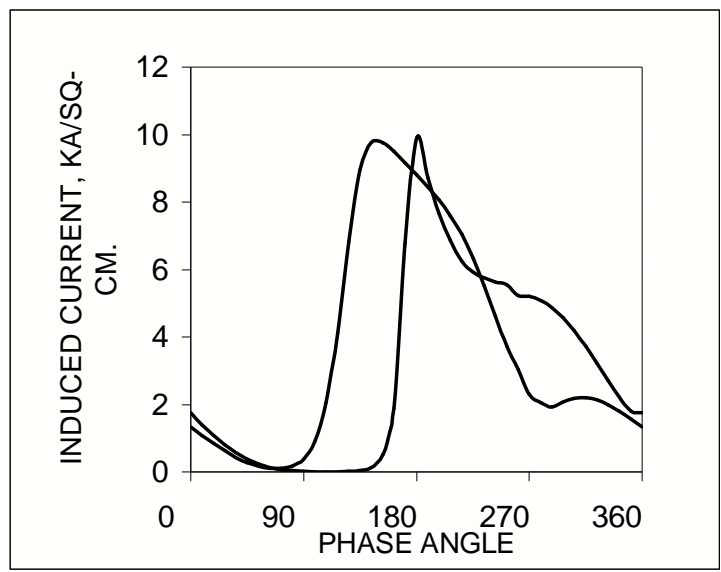

Fig. 6C The induced current for $V_{F}$ and $V_{C}$ at $J_{D C}=3.8$ $\mathrm{KA} / \mathrm{cm}^{2}$

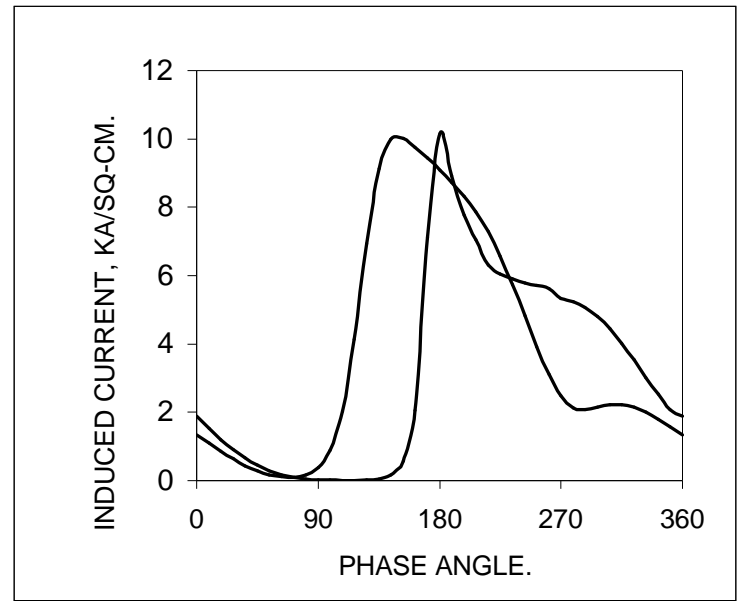

Fig. 6D The induced current for $V_{F}$ and $V_{C}$ at $J_{D C}=3.9$ $\mathrm{KA} / \mathrm{cm}^{2}$

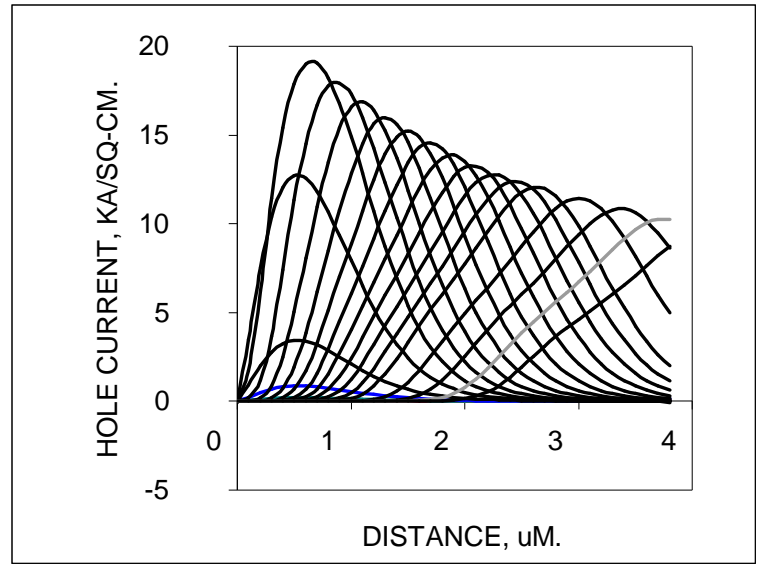

Fig. 7A The hole current versus distance for VF at JDC $=3.5$ $\mathrm{KA} / \mathrm{cm} 2$

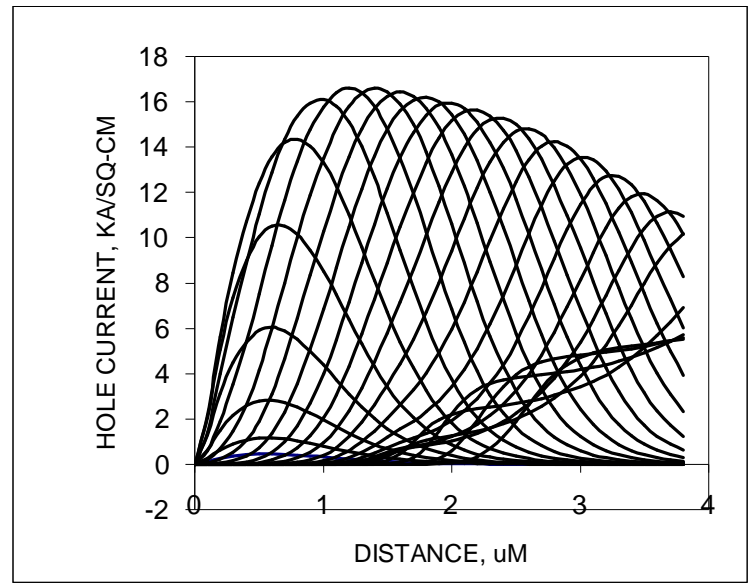

Fig. 7B The hole current versus distance for VC at JDC = $3.5 \mathrm{KA} / \mathrm{cm} 2$

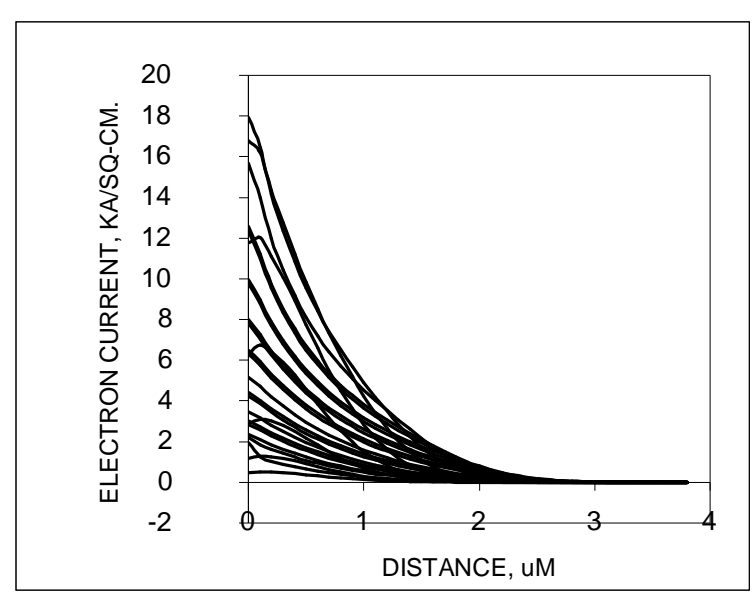

Fig. 8A The electron current versus distance for VC at JDC = $3.5 \mathrm{KA} / \mathrm{cm} 2$ 


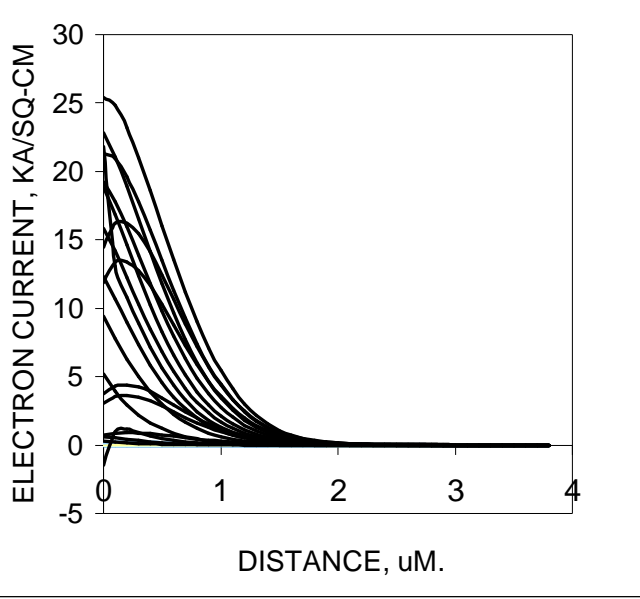

Fig. 8B The electron current versus distance for $\mathrm{V}_{\mathrm{F}}$ at $\mathrm{J}_{\mathrm{DC}}=$ $3.5 \mathrm{KA} / \mathrm{cm}^{2}$

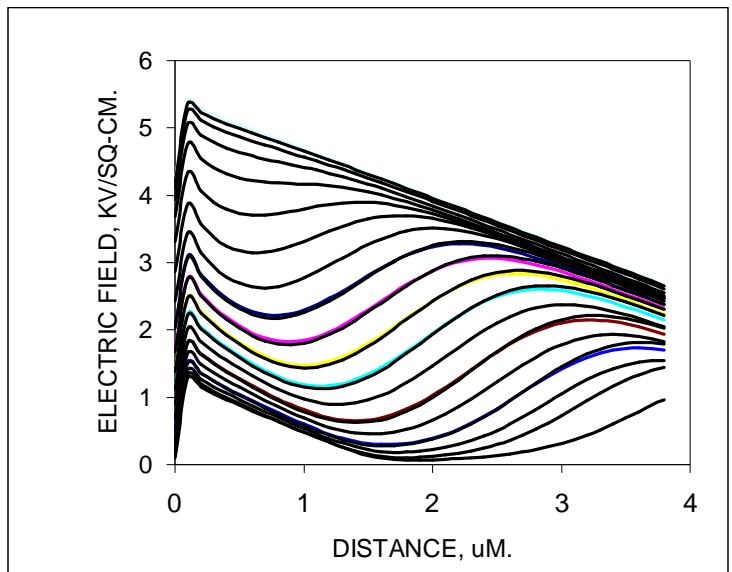

Fig. 9A The electric field versus distance for $V_{C}$ at $J_{D C}=3.5$ $\mathrm{KA} / \mathrm{cm}^{2}$

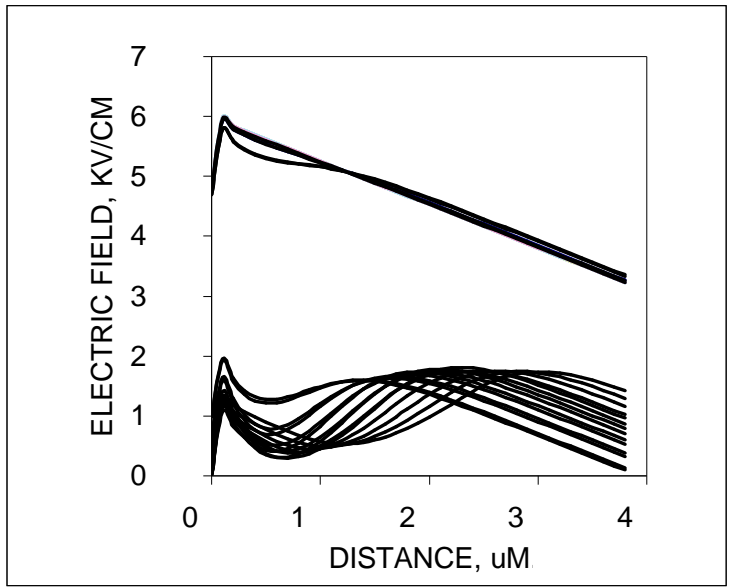

Fig. 9B The electric field versus distance for $V_{F}$ at $J_{D C}=3.5$ $\mathrm{KA} / \mathrm{cm}^{2}$

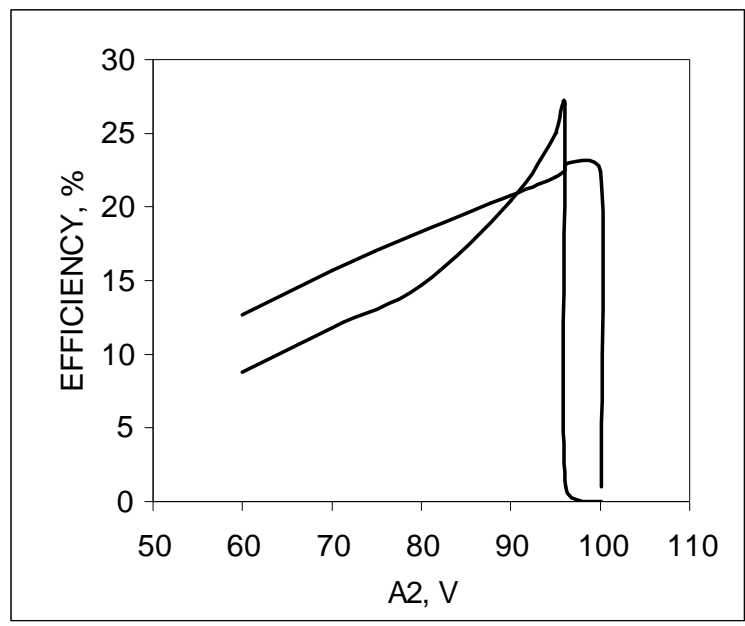

Fig. 10 The efficiency versus $A_{2}$

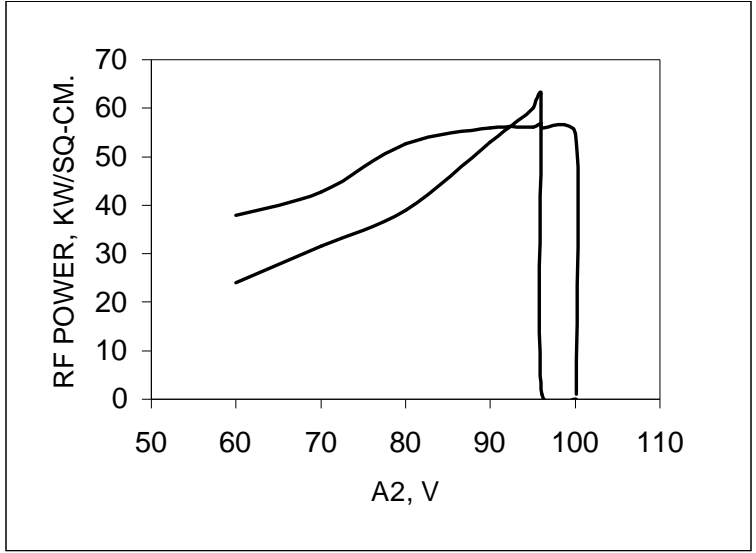

Fig. 11 The RF power versus $A_{2}$

effect and the reduction of the spread of transit times of holes. Since the increase of $A_{2}$ causes $A_{3}$ to increase, the improved performance is attributed also to the reduction of the minority carrier enhancement effect and to the fact that energy extracted in the DR increases with $\mathrm{A}_{2}$ and $\mathrm{A}_{3}$. The saturation and decrease of the power and the efficiency for the large values of $\mathrm{A}_{2}$ are attributed to the drift velocity dropping below saturation and the saturation of the ionization rates at higher electric fields.

\section{THE REASONS LEADING TO THE SUPERIORITY OF THE PROPOSED WAVEFORMS}

The superiority of the performance of the IMPATTs driven by the proposed waveforms is attributed to the following reasons:

1. The considerable reduction of the space charge effect. This is attributed to the high value of the slope of the terminal voltage when crossing the breakdown level and to the large value of this voltage in the time interval during which the avalanche breakdown takes place. 
2. The generation of minority carriers in the drift region is diminished. This is attributed to the higher magnitude of RF voltage during the negative half cycle.

3. The phase delay provided by the avalanche process is increased. This is because both the start of this process and the injection of the AGP in the drift region are delayed. This is attributed to the favorable shape of the proposed waveform and its ability to reduce both the space-charge effect and the minoritycarrier enhancement effect. Therefore, the phase relationship between the terminal voltage and the induced current is improved.

4. The reduction of the spread of transit times of carriers. This is also attributed to the shape of the proposed waveform that helps reduce both the space charge effect and the minority carrier enhancement effect. The proposed waveform causes the carrier generation by the avalanche multiplication to be localized in the time and the space domains.

5. Both the drift-velocity dropping below saturation and the depletion-layer width modulation effects are reduced. This is because of the higher optimum values of the operating dc bias current.

\section{CONCLUSION}

In this paper, the performance of the p-type GaAs IMPATT is studied when it is driven by a proposed non-sinusoidal waveform. It is indicated that by using this waveform, the bad effects that usually degrade the IMPATT performance are reduced. These bad effects include the space-charge effect, the drift-velocity dropping below saturation effect, and the minoritycarrier enhancement effect. It is also illustrated that the proposed waveform helps increase the phase delay provided by the avalanche process and reduce the spread of transit times of carriers. All these factors contribute to the superiority of the proposed waveform over the conventional one. The performance is also studied when the p-type GaAs IMPATT is driven by waveforms consisting only of the first two or three harmonics of the proposed waveform. In this case, the performance of the IMPATT is found superior to that obtained when the device is driven by the conventional waveforms.

\section{REFERENCES}

[1] H. A. El-Motaafy, "A New Physical Explanation for the High-Efficiency Mode of Operation of GaAs IMPATT Diodes", Seventh Conference on Solid-State Science, Cairo, April, 1984.

[2] H. A. El-Motaafy, "The Modes of Operations of GaAs IMPATT Diodes," Proceeding of the Third National Radio-Science Symposium, Cairo, Feb. 1985.

[3] P. Bauhahn and G. I. Haddad, “ IMPATT Device Simulation and Properties", IEEE Trans. Electron Devices, vol. ED-24, Jan., 1977.

[4] K. Mouthan, "Two-Frequency Operation of The Avalanche-Transit-Time Oscillators", Proc. IEEE (Lett.), vol. 58, March 1970.
[5] W. E. Schroeder and G. I. Haddad, "Effect of Harmonic and Subharmonic Signals on Avalanche Diode Oscillator Performance", IEEE Trans. Microwave Theory Tech., vol. MTT-18, June, 1970.

[6] H. El-Motaafy, "The Optimum Voltage for Si-IMPATT Diodes," Seventh Conference on Solid-State Science, Cairo, 1984.

[7] H. El-Motaafy, "Full-Scale Computer Simulation of TRAPATT Diodes, "The Third National Radio-Science Symposium, Cairo, 1985.

[8] El-S. A. El-Badawy, S. H. Ibrahim, and H. A. ElMotaafy, "Analysis of Low-High-Low InP IMPATT Reflection Amplifiers", ANTEM'98, Symposium on Antenna and Applied Electromagnetics, Aug. 9-12, 1998, Ottawa, Ontario, Canada.

[9] S. H. Ibrahim, El-S. A. El-Badawy, and H. A. ElMotaafy, "Analysis and Design Considerations of a Proposed Low-High-Low InP Distributed IMPATT Diode", ICM'97, The International Conference on Microelectronics, Oct., 1997, Bandung, Indonesia.

[10] El-S. A. El-Badawy, S. H. IbrahimH, and H. A. ElMotaafy, "Effect of the Variation of the Doping Density on the Performance of Low-High-Low InP IMPATT Diodes", 1977 North American Radio Science Meeting (IEEE AP-s, International Symposium and URSI Radio-science Meeting, July, 1997, Montreal, Canada.

\section{BIOGRAPHY}

Dr. Hosny Ahmed El-Motaafy was born in 1951, at Cairo, Egypt. He got his B.Sc. and M.Sc. degrees in Electronic Engineering in 1974 and 1979, respectively, from MTC (Military Technical College), Cairo, Egypt and his Ph.D. from ENSAE (Ecole Nationale Supérieure de l'Aéronautique et de l'Espace), Toulouse France, in 1982. Dr El-Motaafy research interest is in microwave electronics and active integrated antennas where he has more than 70 publications. Currently, he holds a position of Associate Professor at the "Higher Technological Institute (HTI)", $10^{\text {th }}$ of Ramadan City, Cairo, Egypt. 